\title{
Synthesis and design of integrated-magnetic-circuit transformer for VRM application
}

\author{
R.-T. Chen and Y.-Y. Chen
}

\begin{abstract}
The paper proposes an improved transformer with an integrated magnetic circuit of the push-pull converter. For high-power server-type applications, the push-pull forward converter has been proven to have significantly better performance than the buck converter. This topology also provides a built-in input filter, and thus a smooth input current. All the magnetic components are integrated in a single core. The simulation of integrated magnetic and magnetic-circuit analysis identifies the proposed transformer correctly. Comparison is given of experimental results of discrete and integrated transformers.
\end{abstract}

\section{List of principal symbols}

$D_{s} \quad$ switch-on duty ratio

$T_{s} \quad$ period of one switching cycle

$V_{i} \quad$ input voltage

$V_{o} \quad$ output voltage

$V_{c r} \quad$ voltage across $C_{r}$

$V_{L r n} \quad$ voltage across $L_{r n}, n=1,2$

$V_{t p n}$ voltage across primary of transformer $\mathrm{TR}_{n}$ $\left(V_{t p n}=V_{p}\right), n=1,2$

$V_{t s n} \quad$ voltage across secondary of transformer $\mathrm{TR}_{n}$ $\left(V_{t s n}=V_{s}\right), n=1,2$

$V_{g n} \quad$ gate voltage of the switch $\mathrm{SW}_{n}, n=1,2$

$V_{p} \quad$ primary voltage of transformer

$V_{s} \quad$ secondary voltage of transformer

$V_{\text {loss }} \quad$ voltage of the stray resistance

$I_{i} \quad$ average input current

$I_{r} \quad$ current through filter $L_{r 2}$

$I_{p} \quad$ sum of the current through $L_{r 1}$ and $L_{r 2}$

$I_{o} \quad$ output average current

$i_{p} \quad$ primary current of transformer

$i_{s} \quad$ secondary current of transformer

$I_{S W n} \quad$ current through switch $\mathrm{SW}_{n}, n=1,2$

$I_{S W n, p k}$ peak current through switch $\mathrm{SW}_{n}, n=1,2$

$I_{L r n, a v} \quad$ average current through $L_{r n}, n=1,2$

$I_{S W n, \text { av }}$ average current through switch $\mathrm{SW} n, n=1,2$

$I_{D n}$, av average current through diode $D_{n}, n=3,4$

$M \quad$ converter voltage gain

$N_{r n} \quad$ the number of turns of the filter inductor $L_{r n}$, $n=1,2$

$N_{p n} \quad$ the number of turns of the primary of transformer

$\mathrm{TR}_{n}\left(N_{p n}=N_{p}\right), n=1,2$

$N_{s n} \quad$ the number of turns of the secondary of transformer $\operatorname{TR}_{n}\left(N_{s n}=N_{s}\right), n=1,2$

$K_{L} \quad$ coefficient: $K_{L}=L_{e q} / R \times T_{s}, L_{e q}=L_{r 1} / L_{r 2}$

(C) The Institution of Engineering and Technology 2006

IEE Proceedings online no. 20050386

doi:10.1049/ip-epa:20050386

Paper first received 20th September 2005 and in final revised form 31st January 2006

The authors are with Department of Electrical Engineering, National Taiwan University No. 1, Sec. 4, Roosevelt Road, Taipei, Taiwan 106, ROC

E-mail: rtchen@ipmc.ee.ntu.edu.tw $k \quad$ coupling coefficient

$\lambda p \quad$ primary flux links of transformer

$\lambda s \quad$ secondary flux links of transformer

$\phi_{T P D} \quad$ flux through transformer for discrete magnetics

$\phi_{L D} \quad$ flux through inductor for discrete magnetics

$\phi_{T P I} \quad$ flux through primary of transformer for integrated magnetics

$\phi_{L I} \quad$ flux through inductor for integrated magnetics

$\phi_{T S I}$ flux through secondary of transformer for integrated magnetics

$L_{e x} \quad$ external inductance of couple inductor

$L_{r M} \quad$ mutual inductance of couple inductor

$L_{r 1} \quad$ primary inductance of couple inductor

$L_{r 2} \quad$ secondary inductance of couple inductor

$M_{t} \quad$ mutual inductance of transformer

$L_{t p} \quad$ primary inductance of transformer

$L_{t s} \quad$ secondary inductance of transformer

$L_{t c} \quad$ outer-core inductance

$L_{t g} \quad$ air gap inductance

$\Re_{t c} \quad$ reluctance of the outer legs

$\Re_{t g} \quad$ reluctance of the airgap leg

$P_{t g} \quad$ permeance of the airgap leg

$P_{t c} \quad$ permeance of the outer cores

$R_{d s(o n)} \quad$ switch MOSFET conduction resistance

$\Delta B$ flux density of transformer

$V_{L} \quad$ inductor equivalent-series-resistance voltage

$\Delta A_{e} \quad$ minimum areas of the core

$n \quad$ turns ratio of the transformer

\section{Introduction}

As computer processors develop quickly, their power management becomes more and more challenging. To provide the power as fast as possible, a so-called, voltageregulator module (VRM) is put close to the processor. Today's $12 \mathrm{~V}$ VRMs usually use multiphase interleaving synchronous-buck topology [1]. Owing to the very low output voltage, the buck converter has a very small duty cycle. This extreme duty cycle impairs the VRM's efficiency and poses obstacles for transient response. With a properly designed turns ratio, the push-pull converter has a 
favourable duty cycle, which provides an opportunity to improve both the efficiency and the transient response [2].

For single-phase-electronics applications, passive power filters and active one- and two-stage rectifiers are typical approaches used to achieve high power factor and low total harmonic distortion (THD). Compared with the two-stage approach, many strategies have been developed to reduce the size and cost and to improve the efficiency. To improve efficiency and reduce size, this paper proposes an improved push-pull converter with an integrated-magnetic-circuit transformer. In this structure, all the magnetic components including input inductor, input-filter inductor and stepdown transformer are integrated into a single EI core. The proposed integrated-magnetic-circuit transformer has a simple core structure, a small leakage inductance and low core losses. A prototype was built to demonstrate the theoretical prediction.

The single-stage DC-DC converter as shown in Fig. $1 a$ integrates a ripple-free input-current shaper and an isolated push-pull converter with two shared switches and controllers. If the duty cycles of switches $\mathrm{SW}_{1}$ and $\mathrm{SW}_{2}$ are made variable and always greater than $50 \%$, i.e. if switches $\mathrm{SW}_{1}$ and $\mathrm{SW}_{2}$ have overlapping conduction intervals, then it operates as boost-type converter and achieves inputcurrent-ripple reduction. One of the advantages of the overlapping primary-switch conduction is the equal division of inductor current between switches $\mathrm{SW}_{1}$ and $\mathrm{SW}_{2}$, thus reducing switch stress and improving conversion efficiency. The proposed push-pull boost-type converter, as shown in Fig. $1 a$, with a duty cycle greater than $50 \%$ is a suitable approach for ripple-free input current. The proposed improved push-pull forward converter, has only a single core for all the magnetic components. The transformer's primary and secondary windings are wound on the two outer legs. The centre leg is utilised for the input filter which includes the leakage inductance of the transformer. Only the centre leg has an airgap. The flux ripple is cancelled at the centre leg, thus increasing the efficiency. This topology is essentially a clamping circuit and a coupled-inductor version of the current shaper. We can clamp the voltage spike and recover the leakage energy. Because of the coupled input inductor, the input-current ripple is greatly reduced, thus increasing the efficiency. The built-in input filter is formed by the leakage inductance between the transformer's primary windings and the clamping capacitor [3]. Because of a near ripple-free input current, the improved push-pull converter has a higher efficiency than the original. This will be verified in the following Sections.

\section{New integrated-magnetic-circuit design}

In this Section a novel magnetic-integration approach is presented for the push-pull boost-type converter. The discrete magnetic circuit of the converter is first designed to let it be operate normally and possess good performance. Then the integrated magnetic circuit is derived, and is employed to find the flux parameters systematically and quantitatively. The performance of the developed converter is demonstrated by some simulation and measured results.

\subsection{Derivation of integrated magnetic circuits}

From Fig. $1 a$ the magnetic core must include inductive elements such as $L_{r 1}, L_{r 2}$, and transformer $\mathrm{TR}_{1}, \mathrm{TR}_{2}$. To
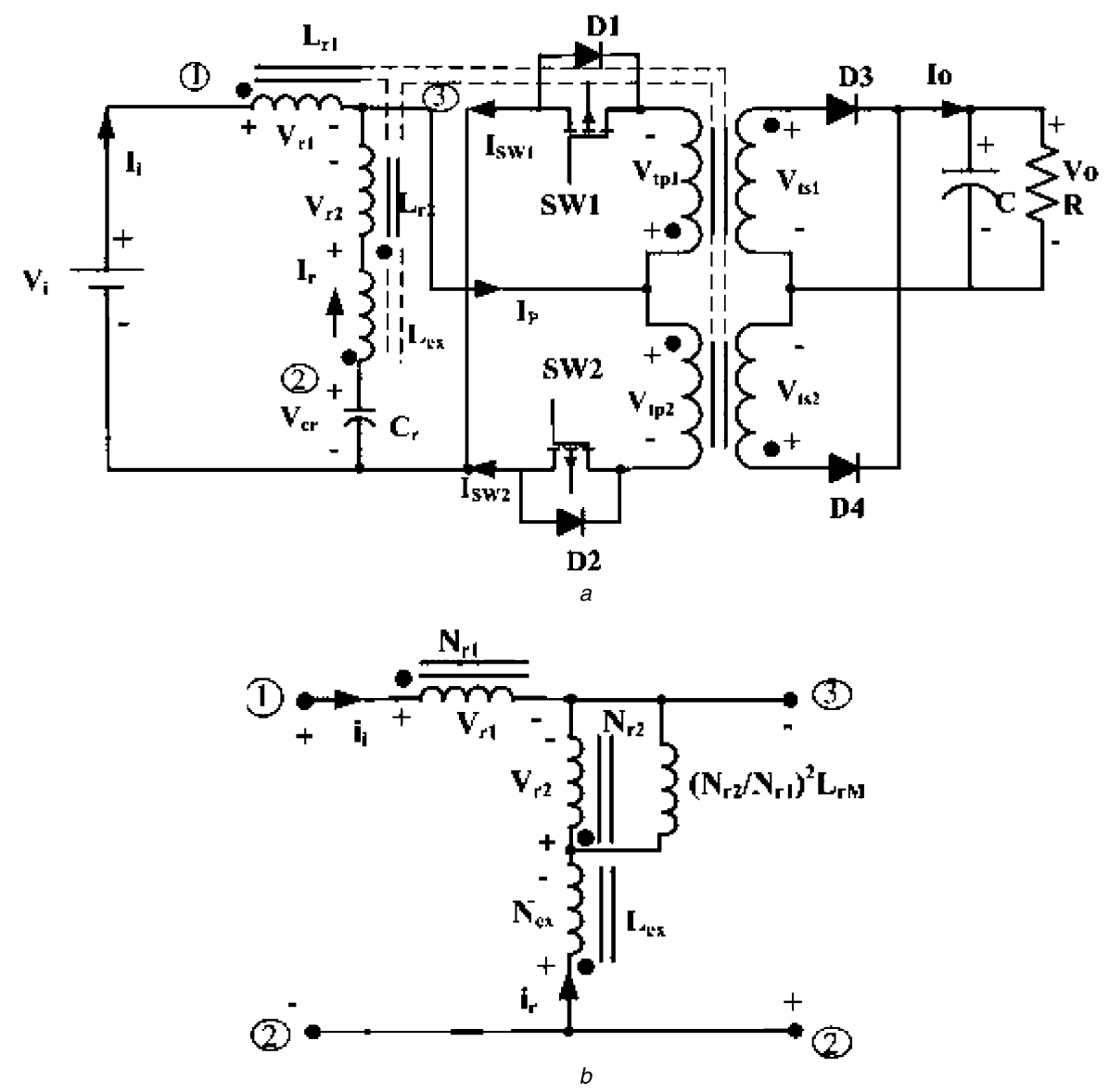

Fig. 1 Proposed push-pull boost-type voltage-regulator module $a$ Power circuit $b$ Two-port representation of the magnetic coupling 


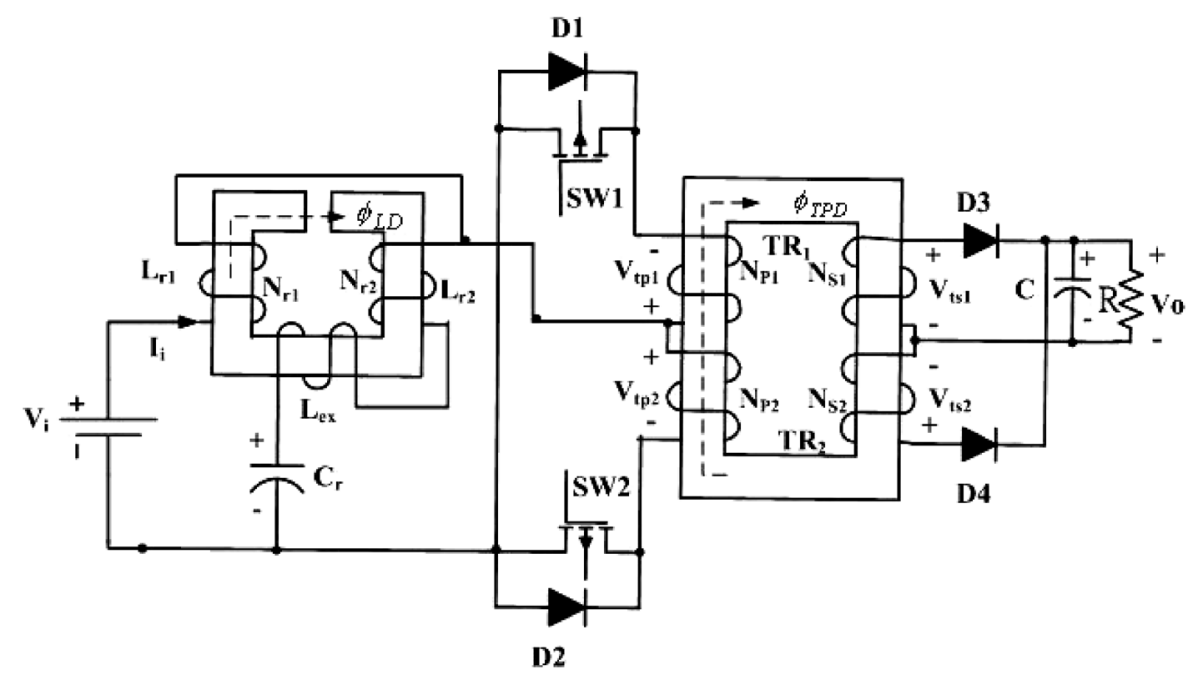

Fig. 2 Schematic of the proposed converter with discrete magnetic core

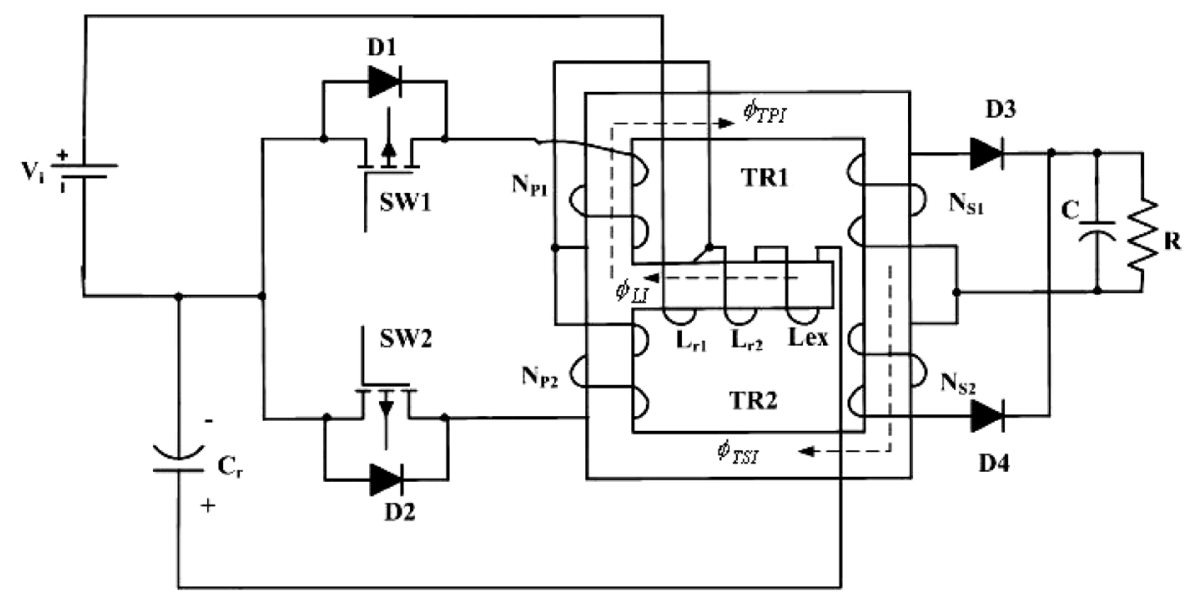

Fig. 3 Schematic of the proposed converter with integrated magnetic core

explain the effected referred to as the zero-ripple phenomenon in [6], we wish to consider briefly the relationships given for magnetic coupling of the two-port networks shown in Fig. $1 b$. It is known that there are several advantages to having $L_{r 1}$ and $L_{r 2}$ wound on the same core. One viable method is to wind primary and secondary turns tightly together to reduce the leakage inductance of $L_{r 1}$ and $L_{r 2}$, to essentially, zero, and then insert a small external trimming inductor in series with the secondary sides to emulate the desired value of $L_{r 2}$. The elimination of the ideal transformer from Fig. $1 a$ results in the model of Fig. $1 b$. Figure 2 shows independent inductor and transformer cores for the magnetic elements. The VRM circuit of Fig. 2 is a discrete magnetic version of the push-pull topologies with transformer isolation. Let us now see if we can formulate an easy way to synthetis the integrated magnetic version of Fig. 3, given the discrete magneticcircuit arrangements as starting points.

To begin, we must first reconstruct the circuit schematics of Fig. 2 so as to detail the magnetic aspects of the transformer and inductor components. A schematic of the result of this reconstruction process is illustrated in Fig. 3. Note that a flux direction within each magnetic component has also been assigned, based on winding polarities produced by converter operation. Next, for each of the switching intervals of the converters, a set of equations defining the rate of change of flux in each magnetic component is established. For this exercise, we can assume all semiconductors of the VRM to be ideal, to simplify our work. Also, we will ignore potential leakage-inductance effects between transformer windings for the same reason, and assume that fluxes are completely contained within its core structure.

Thus, for the converter of Fig. 2 during interval $1\left(\mathrm{SW}_{1}\right.$ and $\mathrm{SW}_{2} \mathrm{ON}$ )

$$
\dot{\phi}_{L D}=\frac{d \phi L D}{d t}=\frac{V_{i}}{N_{r 1}}
$$

where $\phi_{L D}$ is the flux of filter, and $N_{r 1}$ is the number of turns in inductor $L_{r 1}$.

For the converter of Fig. 2 during interval $2\left(\mathrm{SW}_{1} \mathrm{ON}\right.$ and $\mathrm{SW}_{2} \mathrm{OFF}$ ),

$$
\begin{gathered}
\dot{\phi_{L D}}=\frac{d \phi_{L D}}{d t}=\frac{V_{t p 1}}{N_{r 1}}-\frac{V_{i}}{N_{r 1}} \\
\dot{\phi_{T P D}}=\frac{d \phi T P D}{d t}=\frac{V_{o}}{N_{t s 1}}=\frac{V_{t p 1}}{N_{p 1}}
\end{gathered}
$$

where $\phi_{T P D}$ is the flux of the transformer, and $V_{t p 1}, V_{t s 1}$ are the primary and secondary voltages, respectively. We can combine (2) and (3) to eliminate the dependent variable $V_{t p 1}$. Performing this combination gives

$$
\dot{\phi}_{T P D}=\left(\frac{N_{r 1}}{N_{p 1}}\right) \dot{\phi_{L D}}+\frac{V_{i}}{N_{p 1}}
$$

Note that the last term of (4) is of a form that could be considered as defining a flux change in a magnetic medium 
that is dependent on the input voltage $V_{i}$ of the converter and the number of primary turns $N_{p 1}$ on the converter's transformer, Since our goal is to make the inductor a part of the magnetic assembly that contains the transformer component, it is logical to assume that $N_{r 1}$ should be made equal to $N_{p 1}$, so that all of this flux change is contained within one magnetic path or leg of this assembly. Therefore, we arrive at an expression for $\phi_{T P D}$ as

$$
\dot{\phi_{T P D}}=\dot{\phi_{L I}}+\dot{\phi_{T S I}} \equiv \dot{\phi}_{T P I}
$$

We can interpret (5) as defining a magnetic assembly in which there are three major flux paths. It also tells us that the flux change $\phi_{T P I}$ in an input-source-related path contributes to the change in another path associated with the inductor portion of the magnetic assembly $\left(\phi_{L I}\right)$, as well as to flux change in a third path $\left(\phi_{T S I}\right)$. These general observations permit us now to sketch out a magnetic-path arrangement that satisfies the needs of (5). This is done in Fig. 3. It shows the flux distribution in the core. The transformer and the filter inductors in the push-pull converter can be integrated into a single magnetic core. Note that we have added a gap in the inductor-path area because we expect that this leg will have significant DC bias, just as a discrete inductor in a boost converter would experience.

To improve the core structure and to reduce the leakage inductance of the existing integrated magnetics, a novel magnetic-integration approach is proposed in [2]. In this paper, we propose an improved integrated-magnetics core for boost-type push-pull VRM. First, since the transformer's primary winding is split between two outer legs, as shown in Fig. 3 interleaved windings can be used to minimise the leakage inductance of the integrated transformer. Secondly, the polarity of one set of windings is changed through different winding connections. As a result, the direction of flux is also changed. The air gap on the centre leg prevents saturation of the core. The air gap will be the major factors in determining their winding-inductance values, and increasing the air-gap length will decrease the inductance of a magnetic material. Thirdly, as shown in Fig. 3, auxiliary windings can be added to the centre legs of the integrated magnetic converters to reduce input-ripplecurrent magnitudes. The gap inductance $L_{t g}$ is much smaller than that of the ungapped outer legs of the magnetic structure. Both center-leg windings are wound tightly together to maximise magnetic coupling between them and to minimise parasitic leakage inductances.

\subsection{Magnetic-circuit analysis}

A novel magnetic-integration approach is developed for the transformer of the push-pull converter. The flux directions are determined by using right-hand rule, as shown in Fig. 3. From Fig. 3, we assume that, for the double transformer, the numbers of turns of the primary and secondary are the same, i.e. That is, $N_{p 1}=N_{p 2} \equiv N_{p}, N_{S 1}=N_{S 2} \equiv N_{s}$ and $V_{t p 1}=V_{t p 2} \equiv V_{p}, V_{t s 1}=V_{t s 2} \equiv V_{s}$. Additionally, the current may be the same in the ideal circuit, i.e. $i_{p 1}=i_{p 2} \equiv i_{p}$; $i_{s 1}=i_{s 2} \equiv i_{s}$. In the proposed structure, the polarity of one set of windings is changed and, consequently, the direction of $\mathrm{AC}$ flux in one of the two outer legs is also changed. Therefore, the AC fluxes are cancelled in the centre leg. With the reduction of the magnitude of $\mathrm{AC}$ flux in the centre leg, the proposed transformer has a lower core loss in the centre leg. Figure 4 shows the flux distribution in the core for the proposed transformer by using ANSOFT Maxwell simulations. When the inductor acts only as shown in Fig. $4 a$, it reveals that it cannot influence the outer legs.

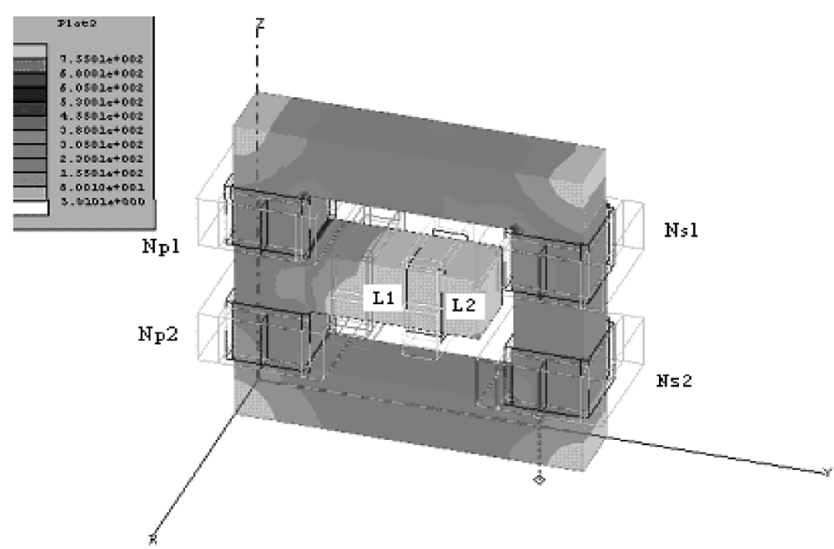

a
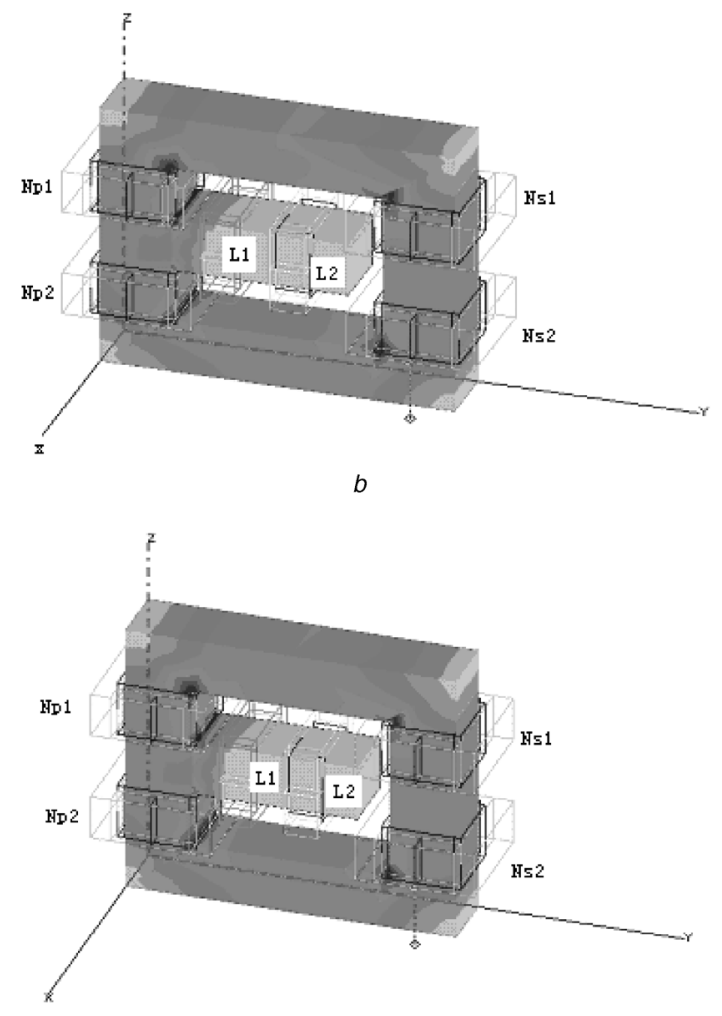

Fig. 4 Simulation of the core-flux distributions of the proposed transformer

$a$ Inject inductor current only

$b$ Inject primary and secondary current only

$c$ Inject normal inductor and transformer current

Figure $4 b$ shows the flux distributions when the transformer injects primary and secondary current only, and Fig. $4 c$ shows the flux distributions when the transformer and inductor inject rated current in normal operation. The simulation results of Fig. $4 b$ and Fig. $4 c$ reveal approximate flux distributions. The outer legs are averaging, distributed in the core and larger than the centre leg. It is shown that fluxes are distributed on the outside equally and that the inner legs relatively small where it is used for the inductor core. This satisfies the former description.

Figure $5 a$ shows the reluctance model for the proposed magnetic circuit. The electrical-circuit model can be derived from the reluctance model by using the principle of duality, as shown in Fig. $5 b$ where $P_{t g}$ is the gap permeance and $P_{t c}$ is the outer-core permeance. The circuit of Fig. $5 c$ results from a scaling step with $N_{p}$ designated as the reference winding. The scaled permeances are then replaced by inductances. From the reluctance circuit shown in Fig. 5a, 

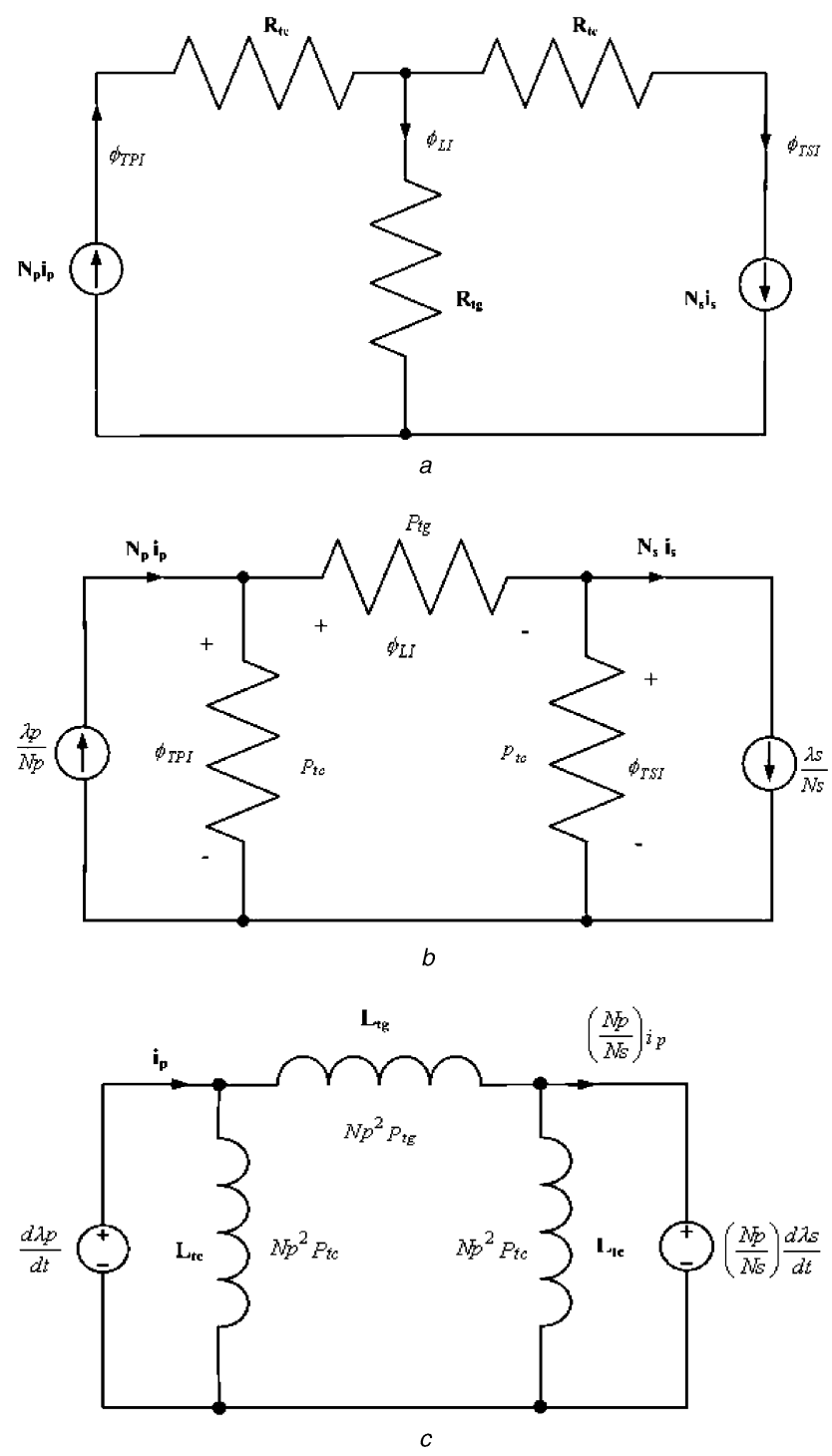

Fig. 5 Model circuits

$a$ Reluctance model of proposed magnetic circuit $b$ Model $(a)$ using the principle of duality

$c$ Model (b) of scaling step with $N_{p}$ designated as reference winding

the fluxes in the cores can be derived in the form of reluctances and MMF sources, as follows [6]:

$$
\begin{aligned}
& \phi_{T P I}=\frac{\Re_{t c}+\Re_{t g}}{\Re_{t c}\left(\Re_{t c}+2 \Re_{t g}\right)} N_{p} \times i_{p}-\frac{\Re_{t g}}{\Re_{t c}\left(\Re_{t c}+2 \Re_{t g}\right)} N_{s} \times i_{s} \\
& \phi_{T S I}=\frac{\Re_{t c}+\Re_{t g}}{\Re_{t c}\left(\Re_{t c}+2 \Re_{t g}\right)} N_{s} \times i_{s}-\frac{\Re_{t g}}{\Re_{t c}\left(\Re_{t c}+2 \Re_{t g}\right)} N_{p} \times i_{p}
\end{aligned}
$$

where $\phi_{T P I}$ and $\phi_{T S I}$ are the fluxes of the primary and secondary cores, $\Re_{t c}$ and $\Re_{t q}$ represent the reluctances of the outer and centre legs, and $i_{p}$ and $i_{s}$ are the total winding currents reflected to the primary and secondary of the transformer, respectively. According to Farady's law, and assuming that the mutual inductance between primary inductance $L_{t p}$ and secondary inductance $L_{t s}$ is $M_{t}$, and the coupling coefficient is $k$, the relationship between inductances and reluctances can be derived as follows:

$$
L_{t p}=\frac{\left(\Re_{t c}+\Re_{t g}\right) N_{p}^{2}}{\Re_{t c}\left(\Re_{t c}+2 \Re_{t g}\right)} \simeq \frac{N_{p}^{2}}{2 \Re_{t c}}
$$

$$
\begin{gathered}
L_{t s}=\frac{\left(\Re_{t c}+\Re_{t g}\right) N_{s}^{2}}{\Re_{t c}\left(\Re_{t c}+2 \Re_{t g}\right)} \simeq \frac{N_{s}^{2}}{2 \Re_{t c}} \\
M_{t}=\frac{\Re_{t g} N_{p} N_{s}}{\Re_{t c}\left(\Re_{t c}+2 \Re_{t g}\right)} \simeq \frac{N_{p} N_{s}}{2 \Re_{t c}} \\
k=\frac{\Re_{t g}}{\Re_{t c}+\Re_{t g}} \simeq 1
\end{gathered}
$$

Similarly, from the reluctance circuit shown in Fig. $5 c$,

$$
\begin{gathered}
L_{t p}=L_{t s}=\frac{L_{t g} L_{t c}+L_{t c}^{2}}{L_{t g}+2 L_{t c}} \simeq \frac{L_{t c}}{2} \\
M_{t}=\frac{L_{t c}^{2}}{L_{t g}+2 L_{t c}} \simeq \frac{L_{t c}}{2}
\end{gathered}
$$

where $L_{t g}=N_{p}^{2} P_{t g}$ is the gap inductance and $L_{t c}=N_{p}^{2} P_{t c}$ is the outer-core inductance.

Since the reluctances from the magnetic material are much smaller than that of the airgap, the reluctance in the centre leg is much larger than those of the outer legs $\left(\Re_{t g} \gg \Re_{t c}\right)$. As can be seen from (8)-(11), in the proposed integrated magnetic structure, the coupling coefficient for the transformer is close to one. Two coupled windings still have a certain amount of leakage inductance. In practice, the reluctances from the magnetic material influence the values of $\Re_{t c}$ and $\Re_{t g}$. Figure 6 shows the equivalent electrical circuit for the proposed integrated magnetic circuit.

\section{Analysis of the proposed VRM}

This Section mainly discusses considerations of transformer design and the steady-state characteristics of the push-pull boost-type VRM. It shows that the proposed boost converter has the same steady-state properties as the conventional boost converter. The prototype was built to demonstrate the theoretical prediction. Some simulations are provided to demonstrate the performance of the developed proposed converter.

\subsection{Design of proposed transformer}

Good transformer design is vital to achieve favourable performance, and is based on the tradeoff between outputcurrent ripple, flux density and magnetic-core size. The transformer core is typical of that used, consisting of some ferrite or powdered-iron materials, for a switching power supply. Commercial magnetic cores are available for selection. In the proposed transformer, we select the TDK core material $\mathrm{H} 7 \mathrm{C} 4$ which is suitable for operation at frequencies over $500 \mathrm{kHz}$. The material $\mathrm{H} 7 \mathrm{C} 4$ has low losses at the required frequency, flux density and operation temperature. From Faraday's electromagnetic law, we can derive the following relation:

$$
V_{P} \times D_{s} \times 10^{4}=2 \times f_{s} \times N_{P} \times \Delta B \times \Delta A_{e}
$$

Where $\Delta B$ is in the tesla and $A_{\mathrm{e}}$ is in the square centimetres, $D_{s}$ is duty ratio, $f_{s}$ is switching frequency, $\Delta B$ is flux density and $\Delta A_{e}$ is the minimum area of the core. According to (14) and the TDK data sheet, the transformercore loss is decided by flux density and core area. Then the transformer-core type will be selected for this application. 


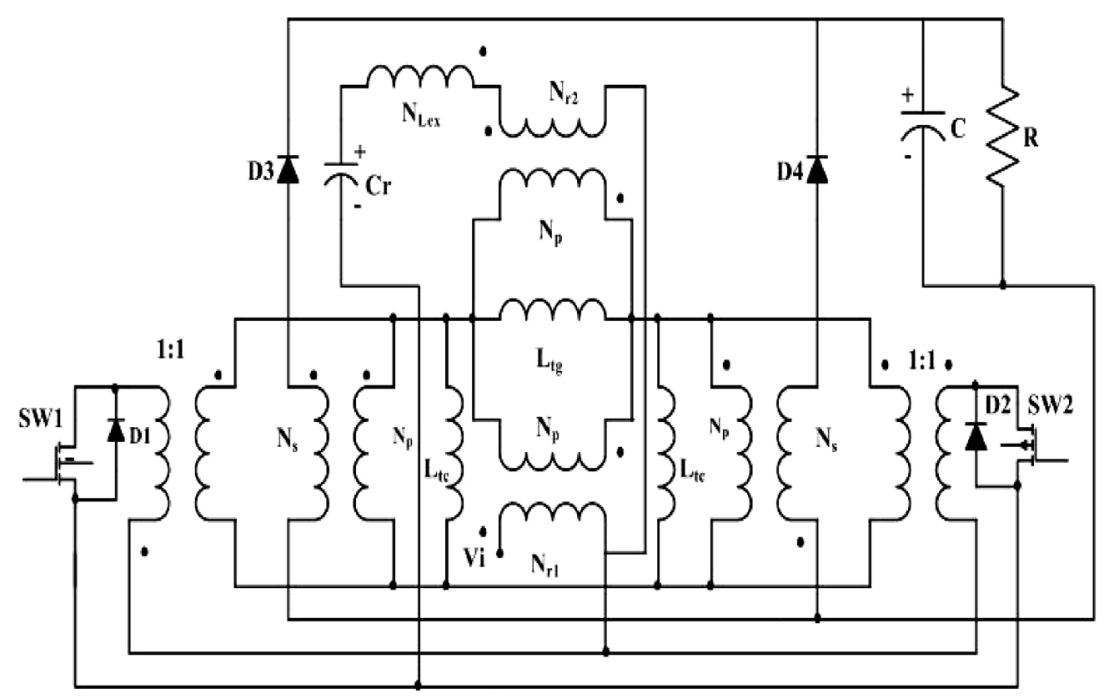

Fig. 6 Equivalent electrical circuit for the proposed integrated magnetic circuits

The transformer turns ratio $n$ can be derived as follows. From the power balance, the primary current $I_{p}$ is

$$
I_{P}=\frac{P_{o}}{\eta D_{s} V_{i}}
$$

where $P_{o}$ is output power, $\eta$ is efficiency and $V_{i}$ is input voltage.

The primary voltage $V_{p}$ and secondary voltage $V_{s}$ of the transformer as shown in Fig. 3 are

$$
\begin{gathered}
V_{P}=V_{i}-V_{L}-I_{P} R_{d s(o n)} \\
V_{S}=V_{o}+V_{D}+V_{\text {loss }}
\end{gathered}
$$

where $V_{L}$ is the inductor equivalent-series-resistance voltage, $I_{p} R_{d s(o n)}$ is the switch MOSFET conduction voltage, $V_{D}$ is the rectifier-diode voltage and $V_{\text {loss }}$ is the voltage of stray resistance loss. Thus, transformer turns ratio is

$$
n=\frac{V_{P} D_{s}}{V_{S}}
$$

According to previous computation, the transformer parameters can be selected to satisfy the electrical and magnetic specifications. The design may follow three steps:

(i) Select the primary-to-secondary winding turns ratio $n$.

(ii) Select the magnetic core.

(iii) Select the winding turns.

In this paper implementation of the proposed transformer will follow these steps, then we will use to select the number of turns of the external inductor for input-current ripple-free consideration.

First, we wish to consider briefly the relationships given for magnetic coupling of the two-port networks shown in Fig. $1 a$. The two-ports network is part of Fig. $1 a$, and the nodes are indicated by (1), (2), and (3). From Fig. $1 b$ the equivalent circuit of the two-port network is represented by an ideal transformer $\left(N_{r 1}: N_{r 2}\right)$ and a mutual inductance $L_{r M}$, where $L_{r M}=\sqrt{ }\left(L_{r 1} L_{r 2}\right)$ under ideal conditions. The elimination of the ideal transformer results in the simple model by transformation from the primary to the secondary side. Then by way of a turns-ratio reflection relation the secondary equivalent inductance is $\left(N_{r 2} / N_{r 1}\right)^{2} L_{r M}$. Let us now make the assumption that it is possible to reduce the value of $i_{i}$ to zero in our model of Fig. $1 a$, and then examine the circuit currents and voltages that result from our assumption. If $i_{i}$ is zero, then the AC-voltage drop across $L_{r 1}$ must also be zero, as illustrated in Fig. $1 a$. The voltage appearing across $L_{r M}$ must therefore be equal to that of the secondary voltage source reflected through the ideal transformer in the model. Also, the current through $L_{r 2}$ and $L_{r M}$ must be equal to that produced by the secondary voltage, $V_{r 2}$ :

These conditions lead to the following simplified relations:

$$
\begin{gathered}
\frac{N_{r 2}}{N_{r 1}} V_{r 1}=L_{r M}\left(\frac{N_{r 2}}{N r_{1}}\right)^{2} \frac{d i_{r}}{d t} \\
V_{r 2}=L_{e x} \frac{d i_{r}}{d t}+L_{r M}\left(\frac{N_{r 2}}{N_{r 1}}\right)^{2} \frac{d i_{r}}{d t}
\end{gathered}
$$

where $i_{r}$ is the current flowing through inductor $L_{r 2}, V_{r 1}$ is the voltage drop between nodes (1) and (3), and $V_{r 2}$ is the voltage drop between nodes (2) and (3).

Combining (19) and (20) by elimination of the common $d i_{r} / d t$ factor gives

$$
L_{e x}=L_{r M}\left(\frac{V_{r 2}}{V_{r 1}}-\frac{N_{r 2}}{N_{r 1}}\right)\left(\frac{N_{r 2}}{N_{r 1}}\right)
$$

where $L_{e x}$ is the external inductance. The magnitude of $L_{e x}$ should satisfy the ripple-free criterion as shown in Fig. $1 b$. The relationship between secondary leakage inductance and that of the core material set by (21) is important and worth dwelling on for a moment. First, recall that this equation was derived based on the premise that no input ripple current existed in our model and, if satisfied, must produce this condition. We can utilise the transformer equations of (21) to select the number of turns of this third winding so as to adjust the VRM's input-current ripple to be essentially zero.

\subsection{Steady-state analysis}

The switching sequences and theoretical waveforms of the proposed converter are illustrated in Fig. 7. The voltages across $L_{r 1}$ and $L_{r 2}$ have the same waveforms and satisfy the ripple-free theoretical condition. The input current $I_{i}$ shows a new steady-state constant value where the ripple variation 


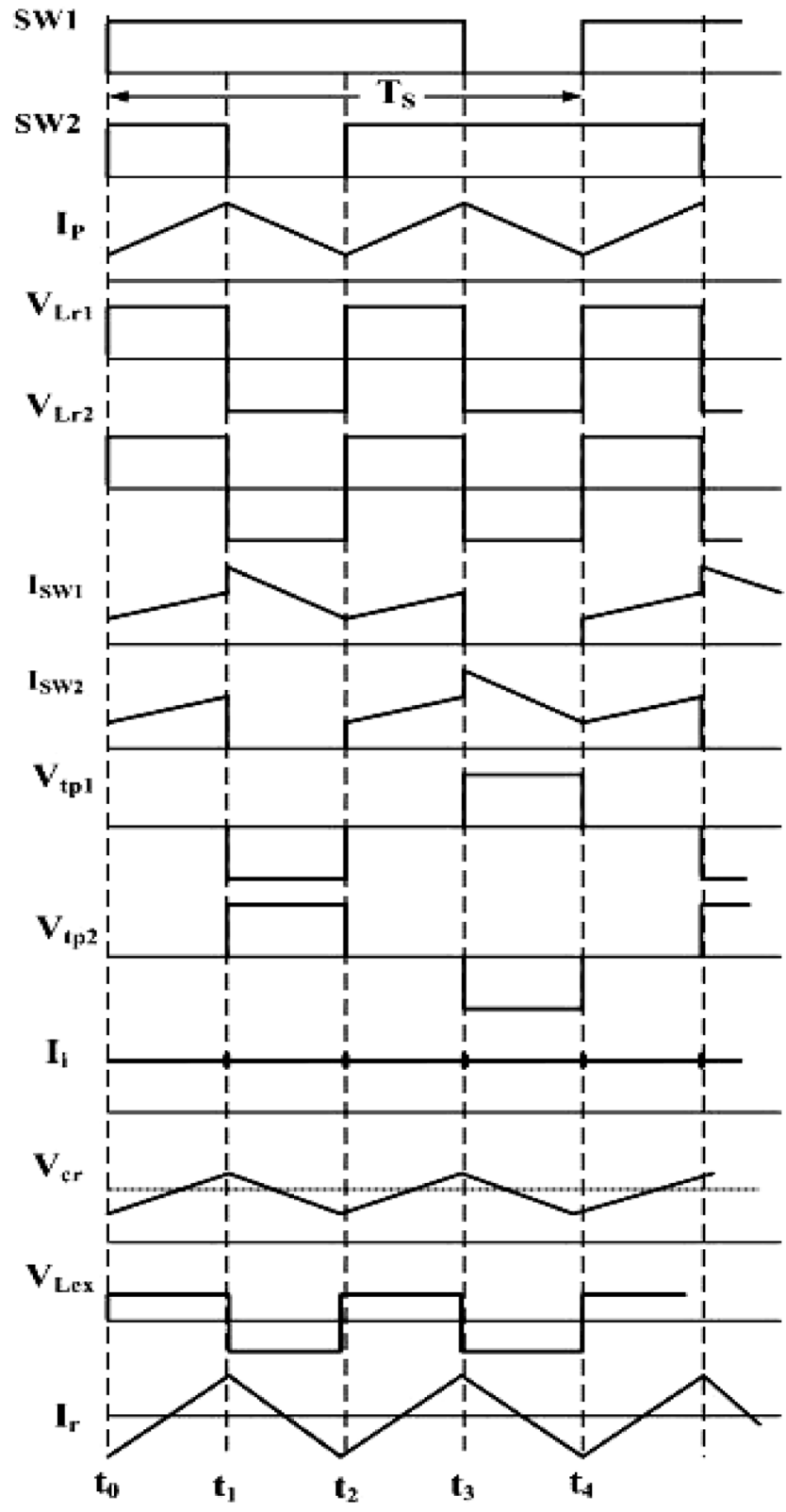

Fig. 7 Main typical waveforms of converter in one switching cycle

is very low. It can be seen that the voltage across capacitor $C_{r}$ is equal to steady-state input voltage $V_{i}$. The voltage across the primary of transformers $\mathrm{TR}_{1}$ and $\mathrm{TR}_{2}$ shows complementary waveforms in half of the switching cycle. As shown in Fig. 7, the switching sequence differs from that of universal push-pull converters. Switches $\mathrm{SW}_{1}$ and $\mathrm{SW}_{2}$ are driven complementary with an overlap interval 1 . The output voltage can be regulated by varying this controllable interval as PWM with a constant switching frequency. The principle of operation in the steady-state condition is described the following assumptions

(i) All the switches and components are ideal.

(ii) Transformers $\mathrm{TR}_{1}$ and $\mathrm{TR}_{2}$ are identical.

(iii) Inductances, $L_{r 1}$ and $L_{r 2}$ are tightly coupled with to each other.

(iv) The output voltage $V_{o}$ is assumed to be constant.

From the switching sequences and theoretical waveforms as shown in Fig. 7, one can identify three intervals in one switching cycle as follows: (a) Interval $1\left(t_{0} \leq t<t_{1}, t_{2} \leq t<t_{3}\right)$ : With switches $\mathrm{SW}_{1}$ and $\mathrm{SW}_{2} \mathrm{ON}$, the inductor $L_{r 1}$ is earth. The input current $I_{i}$ is increased, resulting in energy stored in $L_{r 1}, I_{r}$ is increasing and then changes its direction. Diodes $D_{1}$ and $D_{2}$ are not conducting during this period. The voltage across inductor $L_{r 1}$ is the input voltage $V_{i}$, and the voltage across inductor $L_{r 2}$ is $V_{c r}=V_{i}$.

(b) Interval $2\left(t_{1} \leq t<t_{2}\right)$ : With switches $\mathrm{SW}_{1} \mathrm{ON}$ and $\mathrm{SW}_{2}$ OFF, $I_{r}$ releases the energy stored in $L_{r 1}$ to the transformer $\mathrm{TR}_{1} . I_{r}$ retains its positive direction, however, but is decreasing. The capacitor $C_{r}$ is charged by $I_{r}$. The voltage across inductor $L_{r 1}$ is $\left(V_{i}-V_{p}\right)$. The voltage across inductor $L_{r 2}$ should be $V_{c r}-V_{p}=V_{i}-V_{p}$.

(c) Interval $3\left(t_{3} \leq t<t_{4}\right)$ : With switch $\mathrm{SW}_{1} \mathrm{OFF}$ and switch $\mathrm{SW}_{2} \mathrm{ON}, I_{r}$ releases the energy stored in $L_{r 1}$ to the transformer $\mathrm{TR}_{2}$. The converter action operates as in interval 2.

As a result, the inductances $L_{r 1}$ and $L_{r 2}$ have the same voltage waveforms during the whole cycle. It is possible to couple them and to achieve a reduced component count, reducing the amount of material, increasing the energy density and achieving ripple-free input current [4]. The proposed power converter operated in continuous-conduction mode (CCM) when the duty cycle is always greater than $50 \%$. From the typical waveforms as shown in Fig. 7 the steady-state analysis is derived briefly as follows [7-9]:

According to the voltage-second balance

$$
\left(D_{s}-1 / 2\right) V_{i} T_{s}=\left(1-D_{s}\right)\left(V_{p}-V_{i}\right) T_{s}
$$

where $\frac{V_{o}}{V_{p}}=\frac{N_{s 1}}{N_{p 1}}=\frac{N_{s 2}}{N_{p 2}}=\frac{1}{n}$.

Thus, the following voltage gain can be derived:

$$
M=\frac{V_{o}}{V_{i}}=\frac{1}{2 n\left(1-D_{s}\right)}
$$

From Fig. 7, the average current of $i_{L r 1}$ and $i_{L r 2}$ can be derived as follows:

$$
\begin{aligned}
& I_{L r 1, a v}=\left(\frac{1}{2} D_{s}-\frac{1}{4}\right) \frac{V_{i}}{L_{r 1}} T_{S}+I \\
& I_{L r 2, a v}=\left(\frac{1}{2} D_{s}-\frac{1}{4}\right) \frac{V_{i}}{L_{r 2}} T_{S}-I
\end{aligned}
$$

where $I$ is the steady-state value of the current waveform.

It can be seen that the presence of the capacitor implies that $I_{L r 2, a v}=0$ in this steady state. $L_{e q}$ and $K_{L}$ are defined as follows:

$$
\begin{gathered}
L_{e q}=\frac{L_{r 1} \times L_{r 2}}{L_{r 1}+L_{r 2}} \\
K_{L}=\frac{L_{e q}}{R \times T_{s}}
\end{gathered}
$$

The output average current flows in interval 2 and interval 3 , i.e., when the diode is conducting.

Therefore

$$
I_{o}=\left(1-D_{s}\right)\left(2 D_{s}-1\right) \frac{n V_{i}}{2 L_{e q}} T_{s}
$$

Using the assumption of $100 \%$ efficiency, the average input current $I_{i, a v}$ can be derived

$$
I_{i, a v}=\frac{I_{o} \times V_{o}}{V_{i}}=\left(1-D_{s}\right)\left(2 D_{s}-1\right) \frac{n V_{o}}{2 L_{e q}} T_{s}
$$


The switch average current is

$$
I_{S W 1, a v}=I_{S W 2, a v}=\frac{V_{i} \times T_{s}}{2 L_{e q}}\left(D_{s}-\frac{1}{2}\right)\left(\frac{3}{2}-D_{s}\right)
$$

The diode average current is

$$
I_{D 3, a v}=I_{D 4, a v}=\left(1-D_{s}\right)\left(2 D_{s}-1\right) \frac{n V_{i} \times T_{s}}{2 L_{e q}}
$$

The switch peak current is

$$
i_{S W 1, p k}=I_{S W 2, p k}=\left(2 D_{s}-1\right) \frac{V_{i} \times T_{s}}{2 L_{e q}}
$$

Based on the previous analysis, Fig. 8 shows some characteristic curves for the proposed converter. It shows that the proposed boost-type converter has the same steady-

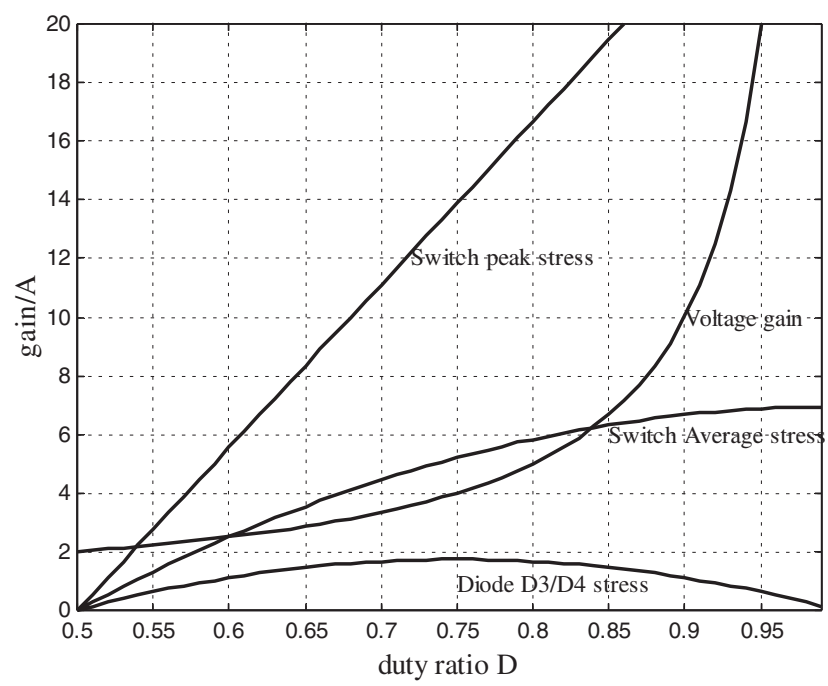

Fig. 8 Characteristic curves for the proposed converter state properties as the conventional boost converter. It can achieve continuous input current with a duty cycle greater than $50 \%$. The improved push-pull converter appears to give lower switch stress and higher efficiency than conventional types.

\section{Experiment and results}

The new push-pull converter was implemented as shown in Fig. 9, with the following specifications: output power $P_{o}=80 \mathrm{~W}$; input voltage $V_{i}=44-52 \mathrm{~V}$; output voltage $V_{o}=1-5 \mathrm{~V}$; switching frequency $f_{s}=150 \mathrm{kHz}$. The power stage consists of the following components: switches $\mathrm{SW}_{1}$ and $\mathrm{SW}_{2}$ : power MOSFETs IRF 740; diodes $\mathrm{D}_{1}$ and $\mathrm{D}_{2}$ : HFA08TB60; diodes $\mathrm{D}_{3}$ and $\mathrm{D}_{4}$ : HFA15TB40; capacitor $C_{r}: 10 \mu \mathrm{F}$; couple inductor $L_{r 1}=L_{r 2}=6.4 \mu \mathrm{H}$; extra inductor $L_{e x}=1.4 \mu \mathrm{H}$; core: TDK EI $35 ; N_{p 1}=N_{p 2}=96$ turns; $N_{s 1}=N_{s 2}=12$ turns; $N_{r 1}=N_{r 2}=36$ turns; $N_{e x}=8$ turns; output capacitor $C: 3300 \mu \mathrm{F}$.

The switch-conduction sequences of the proposed converter are shown in Fig. 10a. It shows that the duty cycles are greater than $50 \%$. Thus, the proposed push-pull converter works in symmetrical continuous-conduction boost mode. The input-current shaper will achieve a low ripple in this situation. The experimental MOSFET switches' drain-to-source-voltage waveforms are shown in Fig. 10b, which shows that the switch stress is reduced and the switching loss may benefit from this. The input-current shaper achieves low ripple in this situation. The voltages across the primaries of transformers $\mathrm{TR}_{1} \mathrm{TR}_{2}$ are shown in Fig. 11. The results are consistent with the typical waveforms as shown in Fig. 7.

To demonstrate the theoretical prediction, the proposed VRM with and without input-current filter were implemented. Figure 12 shows the experimental input-voltage waveforms with the integrated and discrete magnetic-circuit VRM with and without filter, respectively. From Fig. 12c, it can be seen that the filter provides better ripple reduction. The harmonic of the converter without a filter is the worst

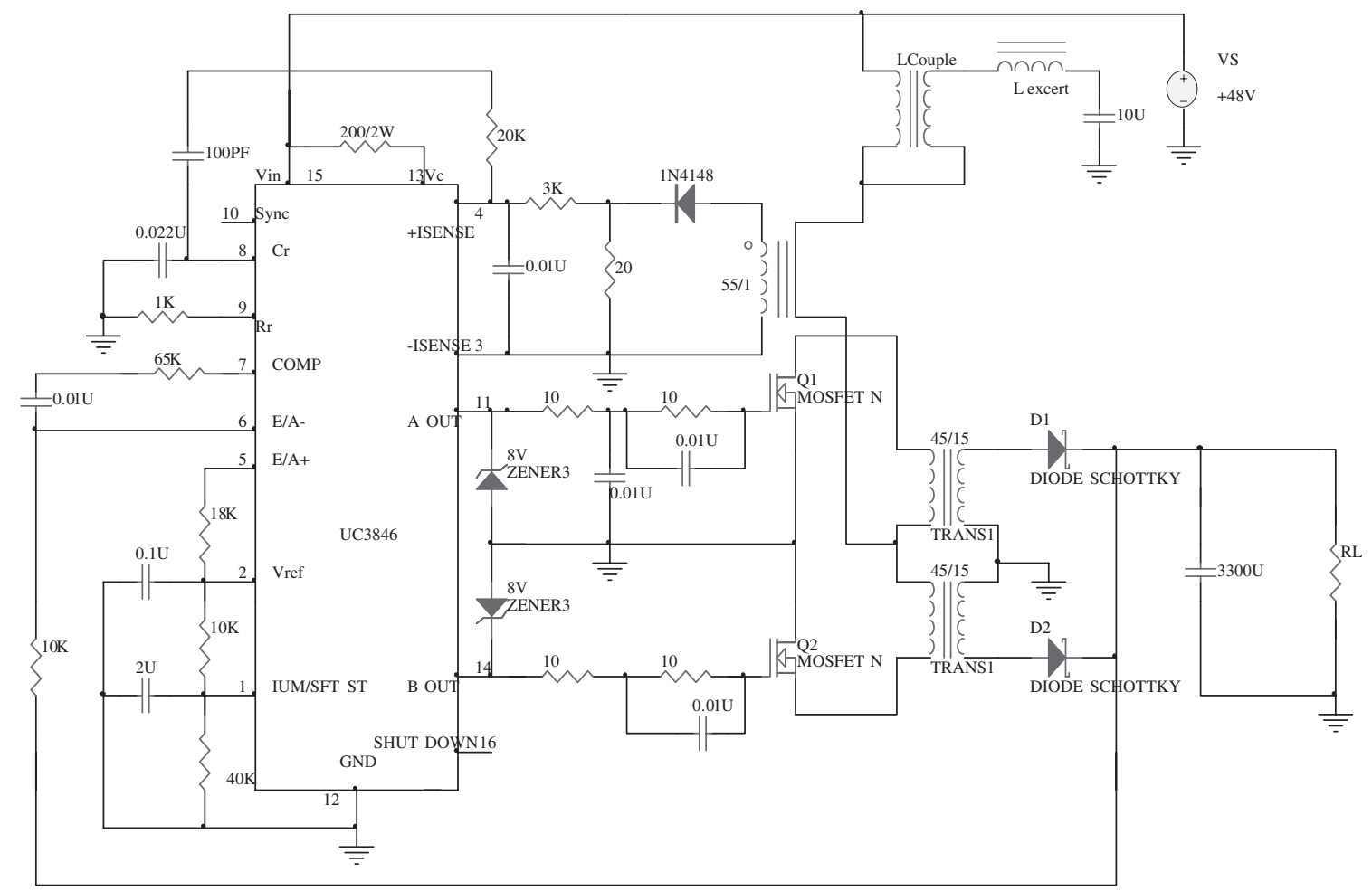

Fig. 9 Schematic of the proposed converter 

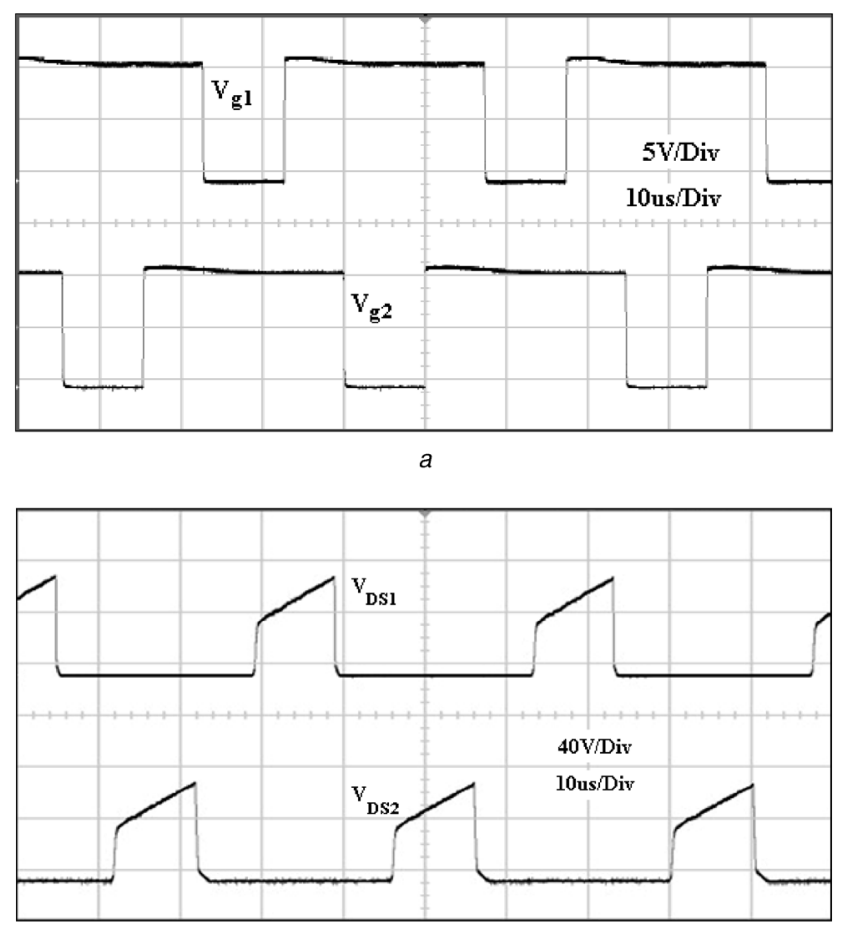

Fig. 10 Measured switch MOSFET waveforms

$a$ Gate voltage $\left(V_{g 1}, V_{g 2}\right)$

$b$ Drain to source voltage $\left(V_{D S 1}, V_{D S 2}\right)$

Voltage: $5 \mathrm{~V} /$ division

Time: $10 \mu \mathrm{s} /$ division

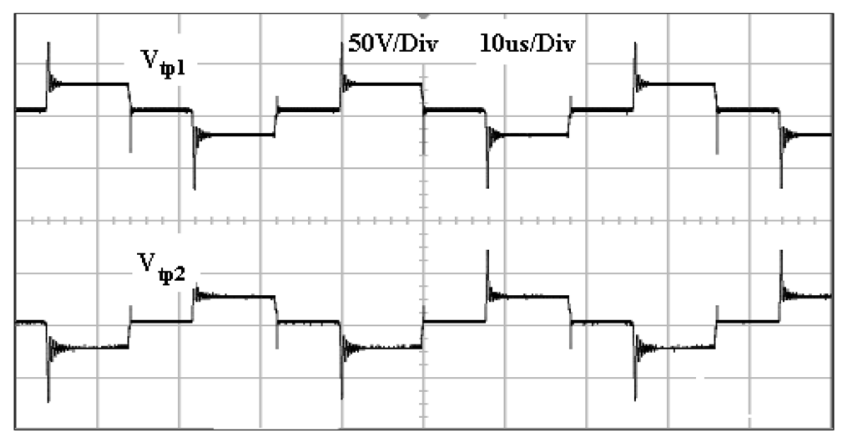

Fig. 11 Experiment results: voltages across primary of transfor$\operatorname{mer}\left(V_{t p 1}, V_{t p 2}\right)$

Voltage: $50 \mathrm{~V} /$ division

Time: $10 \mu \mathrm{s} /$ division

case. However, the harmonics of the proposed converter with integrated and discrete magnetic circuits are approximately the same. They also satisfy the prediction of better low-ripple.

The experimental results for the transformer core loss and total weight with respect to output power are shown in Figs. 13 and 14 for discrete and integrated cases, respectively. Figure 13 shows that the core loss for a discrete magnetic circuit under a heavy load will lower than that for the integrated magnetic circuit. From Fig. 14, the discrete magnetic circuit of the converter is the worse case, because the integrated-magnetic-circuit transformer has a simple core structure and a smaller size, weight and lower core losses. Finally, the measured efficiency for the power stage for the three different conditions is shown in Fig. 15. The maximum value at full load is about $92 \%$, and the converter without a filter is the worst case.
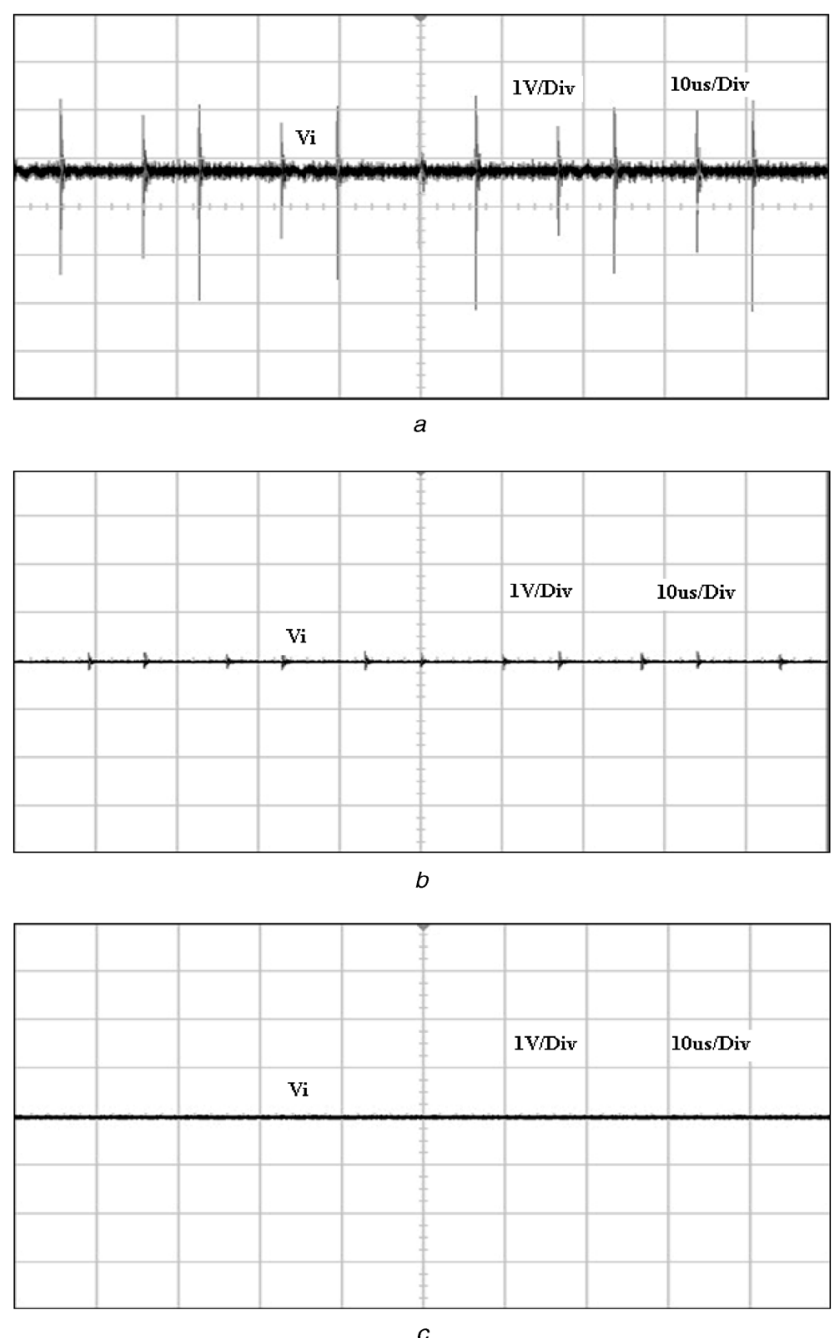

Fig. 12 Measured input voltage $a$ Integrated magnetics without filter $b$ Integrated magnetics with filter $c$ Discrete magnetics with filter Voltage $1 \mathrm{~V} /$ division

Time: $10 \mu \mathrm{s} /$ division

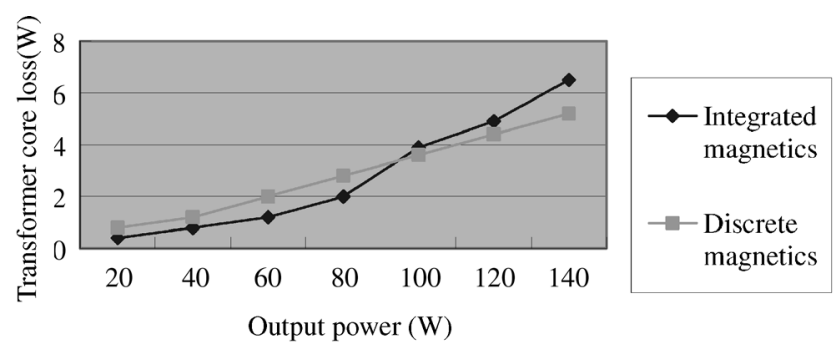

Fig. 13 Comparison of transformer core-loss-experiment results for discrete and integrated magnetic. with respect to output power

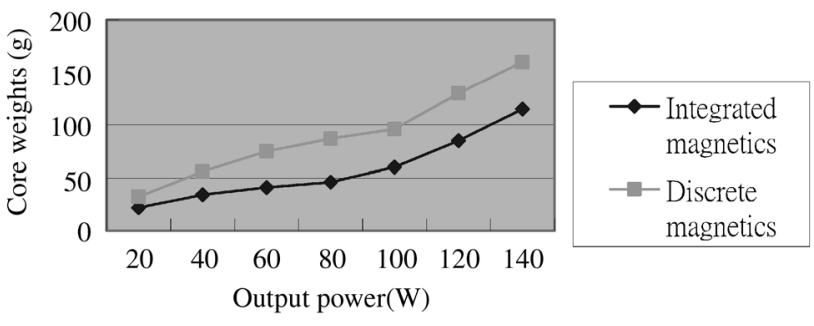

Fig. 14 Comparison of transformer core-weight-experiment results for discrete and integrated magnetics with respect to output power 


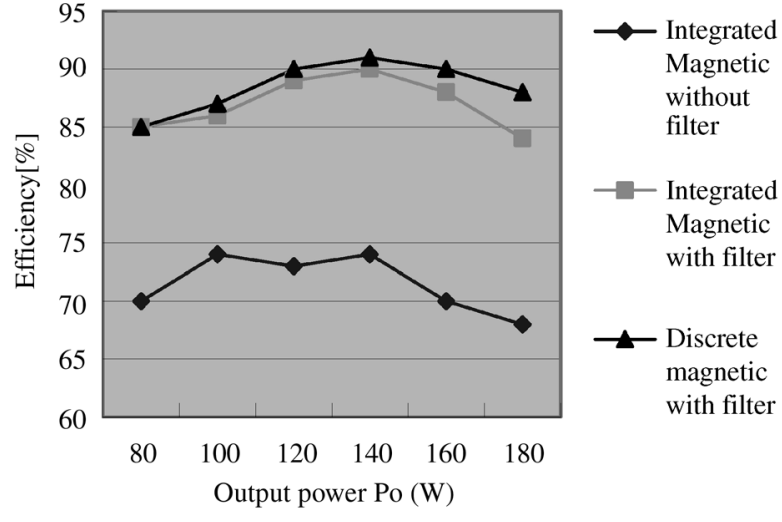

Fig. 15 Comparison of efficiency

\section{Conclusions}

The improved push-pull converter with input-currentshaper is a suitable scheme for high-input VRM design. In the proposed topology, an integrated-magnetic-circuit transformer has been developed for the ripple reduction in order to make the high-input VRM more efficient, more compact and less expensive, where the transformer's primary and secondary windings are wound on the two outer legs. Blending of the inductors and transformers of VRM into single magnetic systems can be very advantageous, often resulting in converter designs of lower cost, weight and size than their discrete-magnetic-circuit counterparts. Finally, the conversion performance can also be improved and component stresses reduced, provided that the integration process is well thought out and properly executed.

\section{References}

1 Zhou, X., Wong, P.-L., Xu, P., Lee, F.C., and Huang, A.Q.: 'Investigation of candidate VRM topologies for future microprocessors', IEEE Trans. Power Electron., 2000, 15, (6), pp. 1172-1182

2 Xu, P., Ye, M., Wong, P.L., and Lee, F.C.: 'Design of $48 \mathrm{~V}$ voltage regulator modules with a novel integrated magnetics', IEEE Trans. Power Electron. 2002 17, pp 990-998

3 Huber, L., Zhang, J., Jovanovic, M.M., and Lee, F.C.: 'Generalised topologies of single-stage input-current-shaping circuits', IEEE Trans. Power Electron., 2001, 16, pp. 508-513

4 Wang, J., Dunford, W.G., and Mauch, K.: 'Analysis of a ripple-free input-current boost converter with discontinuous conduction characteristics', IEEE Trans. Power Electron., 1997, 12, pp. 684-694

5 Garci'a, O., Cobos, J.A., Alou, P., and Uceda, J.: 'A simple singleswitch single-stage $\mathrm{AC} / \mathrm{DC}$ converter with fast output voltage regulation', IEEE Trans. Power Electron., 2002, 17, pp. 163-171

6 Chen, R.T., and Chen, Y.Y.: 'A novel single stage push pull converter with integrated magnetics and ripple-free input current'. Trans. IEEE Power Electronics Specialist Conf., 2004, pp. 3848-3853

7 Zhao, Q. Lee, F.C., and Tsai, F. 'Voltage and current stress reduction in single-stage power factor correction AC/DC converters with bulk capacitor voltage feedback', IEEE Trans. Power Electron., 2002, 17, pp. $477-484$

8 Huber, L., Zhang, J., Jovanovic', M.M., and Lee, F.C.: 'Generalized topologies of single-stage input-current-shaping circuits', IEEE Trans. Power Electron., 2001, 16, pp. 508-513

9 Abu-Qahouq, J., and Batarseh, I.: 'Unified steady-state analysis of softswitching DC-DC converters', IEEE Trans. Power Electron., 2002, 17, pp. $684-691$ 